OPEN ACCESS

Edited by: Aldo Tagliabue,

Italian National Research Council, Italy

Reviewed by:

Georg Varga,

University Hospital Muenster,

Germany

Edward $N$ Harris,

University of Nebraska System,

United States

${ }^{*}$ Correspondence:

Julia Kzhyshkowska

Julia.Kzhyshkowska@medma.uni-

heidelberg.de

${ }^{\dagger}$ These authors have contributed equally to this work

Specialty section:

This article was submitted to Cytokines and Soluble Mediators in Immunity, a section of the journa

Frontiers in Immunology

Received: 18 August 2021 Accepted: 23 November 2021 Published: 15 December 2021

Citation:

Silva-Bermudez LS, Sevastyanova TN, Schmuttermaier C, De La Torre C,

Schumacher L, Klüter $\mathrm{H}$ and Kzhyshkowska J (2021) Titanium Nanoparticles Enhance Production and Suppress Stabilin-1-Mediated

Clearance of GDF-15 in Human Primary Macrophages.

Front. Immunol. 12:760577. doi: 10.3389/fimmu.2021.760577

\section{Titanium Nanoparticles Enhance Production and Suppress Stabilin-1- Mediated Clearance of GDF-15 in Human Primary Macrophages}

\author{
Lina S. Silva-Bermudez ${ }^{1,2 \dagger}$, Tatyana N. Sevastyanova ${ }^{1 \dagger}$, Christina Schmuttermaier ${ }^{1}$, \\ Carolina De La Torre ${ }^{3}$, Leonie Schumacher ${ }^{1}$, Harald Klüter ${ }^{1,2}$ and Julia Kzhyshkowska ${ }^{1,2 *}$ \\ 1 Institute of Transfusion Medicine and Immunology, Medical Faculty Mannheim, Heidelberg University, Mannheim, Germany, \\ ${ }^{2}$ German Red Cross Blood Service Baden-Württemberg - Hessen, Mannheim, Germany, ${ }^{3}$ Microarray Analytics - NGS Core \\ Facility (IKC), Medical Faculty Mannheim, University of Heidelberg, Mannheim, Germany
}

Macrophages are key innate immune cells that mediate implant acceptance or rejection. Titanium implants degrade over time inside the body, which results in the release of implant wear-off particles. Titanium nanoparticles (TiNPS) favor pro-inflammatory macrophage polarization (M1) and lower tolerogenic activation (M2). GDF-15 regulates immune tolerance and fibrosis and is endocytosed by stabilin-1. How TiNPs affect the healing activities of macrophages and their release of circulating cytokines is an open question in regenerative medicine. In this study for the first time, we identified the transcriptional program induced and suppressed by TiNPs in human pro-inflammatory and healing macrophages. Microarray analysis revealed that TiNPs altered the expression of 5098 genes in M1 (IFN- $\gamma$-stimulated) and 4380 genes in M2 (IL-4-stimulated) macrophages. 1980 genes were differentially regulated in both M1 and M2. Affymetrix analysis, confirmed by RT-PCR, demonstrated that TiNPs upregulate expression of GDF15 and suppress stabilin-1, scavenger receptor of GDF-15. TiNPs also significantly stimulated GDF-15 protein secretion in inflammatory and healing macrophages. Flow cytometry demonstrated, that scavenging activity of stabilin-1 was significantly suppressed by TiNPs. Confocal microscopy analysis showed that TiNPs impair internalization of stabilin-1 ligand acLDL and its transport to the endocytic pathway. Our data demonstrate that TiNPs have a dual effect on the GDF-15/stabilin-1 interaction in macrophage system, by increasing the production of GDF-15 and suppressing stabilin-1 mediated clearance function. In summary, this process can result in a significant increase of GDF-15 in the extracellular space and in circulation leading to unbalanced pro-fibrotic reactions and implant complications.

Keywords: titanium, nanoparticle, macrophage, endocytosis, scavenger receptor, growth factor 


\section{INTRODUCTION}

Implantation of biomedical devices is one of the most frequently performed procedures in fields such as orthopedics and dentistry ( 1 , 2). Macrophages play a critical role in the modulation of implant microenvironment (3). Upon implantation, monocytes are recruited from the circulation to differentiate into macrophages and react against the foreign body. The recognition of the material as a foreign body in the tissue encompasses macrophage interaction with the implant surface. This interaction favors the release of chemokines, which recruit additional macrophages and other immune cells leading to acute inflammation (4). Among the many different subtypes of macrophages, there are two activation patterns in which macrophages can be found in vitro, according to their function: pro-inflammatory (M1) or regulatory (M2). M1 differentiation is obtained in response to interferon- $\gamma$ (INF- $\gamma$ ) and lipopolysaccharide and contributes to acute cytotoxic responses. M2 differentiation occurs in response to interleukin 4 (IL-4) or IL13 and it is associated with healing responses, promoting local tissue remodeling (5). M0 are unpolarized or uncommitted macrophages that have neither pro-inflammatory nor anti-inflammatory characteristics, and which differentiation is driven by M-CSF (6). An adequate balance between these subtypes of macrophage influences the degree of osseointegration (7). In contrast, a dysregulated immune response leads to complications, such as infection and aseptic loosening, which are indications for surgical revision $(8,9)$. Implant revision not only negatively affects the patient's quality of life but also aggravates the economic burden due to an increase in hospitalization rates and in the necessity of reintervention (10).

Titanium is a widely used material due to its advantageous biocompatibility properties, corrosion resistance, and low magnetic susceptibility (1). However, with time and friction, it generates wear-off particles, also known as implant debris. Particles of a diameter smaller than $1 \mu \mathrm{m}$, or nanoparticles, generate the most biological toxicity and can induce mutations $(11,12)$. Titanium nanoparticles (TiNPs) are released in vitro, even in the absence of implant friction (13). TiNPs are internalized by macrophages in a dose-dependent matter and promote sustained production of proinflammatory factors, which can result in acute and chronic inflammation (14-17). For instance, lysosomal cathepsins are released, activating nod-like receptor protein 3 (NRLP3) inflammasome, and as a consequence, IL- $1 \beta$ release, promoting osteoclast differentiation and peri-implant osteolysis (18). Pajarinen et al, showed an exacerbated pro-inflammatory profile in human CD14+ derived M1 and a suppressed inflammatory response in M2, in response to co-culturing with TiNPs (19). Supporting this preferential polarization, oxidative stress, mainly mediated by M1 phenotype, is a frequent response to TiNPs (17).

Despite isolated reports about the pro-inflammatory effects of TiNPs on macrophages, no systematic analysis has been performed to date to show the full transcriptional program affected by TiNPs in pro-inflammatory and healing macrophages. In this study for the first time, we addressed the question about the stimulatory and suppressive effect of TiNPs on the transcriptional program in human pro-inflammatory and healing macrophages. We found that TiNPs stimulate all subtypes of macrophages to produce growth differentiation factor 15 (GDF-15), a cytokine involved in the regulation of tissue remodeling, healing, and angiogenesis, with growing evidence about its implication in pathology. GDF-15 is also known for being a multifunctional cytokine mainly expressed and secreted during stress conditions. Although its role is still controversial, GDF-15 is hypothesized to be part of a negative feedback mechanism to counteract inflammatory reactions (20). We also found that TiNPs have a specific suppressing effect on the scavenging function of stabilin-1, which is a clearance receptor of GDF-15. This dual effect can result in the uncontrolled increase of GDF-15 levels in the tissues in close proximity to implants as well as in the circulation of patients with implants.

\section{METHODS}

\section{Monocyte Isolation and Generation of Macrophages}

Monocytes were isolated from Buffy coats obtained from healthy blood donors out of the German Red Cross Blood Service BadenWürttemberg - Hessen after informed consent, as described previously (21). The isolation was carried out using CD14 positive selection (Miltenyi Biotec), resulting in 90-98\% monocyte purity, controlled by flow cytometry. The cells were seeded into cell culture dishes in customized serum-free medium (SFM from Gibco) supplemented with $5 \mathrm{mM}$ glucose at a concentration of $1 \times 10^{6}$ cells/ $\mathrm{mL}$. Macrophage were differentiated in the presence of M-CSF at 5 ng/mL (Peprotech; \#A300-25B) and Dexamethasone $10^{-8} \mathrm{M}$ (Sigma, $\# \mathrm{D} 2915)$. For M1 polarization IFN- $\gamma$ was used at the concentration of $100 \mathrm{ng} / \mathrm{mL}$ (Peprotech; \# 300-02), for M2 polarization, IL-4 was used at the concentration of $10 \mathrm{ng} / \mathrm{mL}$ (Peprotech; \#200-04). No cytokines were added for M0 differentiation.

\section{Stimulation With TiNPs}

Titanium nanoparticles were purchased from NanoAmor Europe, France. The stock solution was initially diluted to 1:4 in DPBS, followed by another 10-fold dilution in $5 \mathrm{mM}$ glucose SFM media to achieve the final dilution factor (1:4000). The TiNPs were added to a final concentration of $0,0100 \%$ (100ppm). The corresponding dilution was sterilized via UV.

The conditions were maintained for 6 days with $7.5 \% \mathrm{CO} 2$ at $37^{\circ} \mathrm{C}$. A daily microscopic check-up was performed to evaluate the health status of the cells. Additionally, the viability of macrophages was assessed using Alamar Blue test.

\section{RNA Isolation and Affymetrix Chip Analysis}

After incubation with 0,0100\% (100ppm) TiNPs for 6 days, cells were lysed in TRK lysis buffer and RNA was isolated using E.Z.N.A. Total RNA kit I (Omega Bio-tek, USA) according to the manufacturer's instructions. The concentration of isolated RNA was determined with a Tecan Infinite ${ }^{\circledR} 200$. RNA was tested by capillary electrophoresis on an Agilent 2100 bioanalyzer (Agilent) and high-quality was confirmed. Hybridization of probes was done using arrays of human HuGene-1_0-st-type (Affymetrix, High Wycombe, UK). Biotinylated antisense cRNA was then prepared according to the Affymetrix standard labeling protocol with the GeneChip ${ }^{\circledR}$ WT Plus Reagent Kit and the 
GeneChip ${ }^{\circledR}$ Hybridization, Wash and Stain Kit (both from Affymetrix, Santa Clara, USA). Afterward, the hybridization on the chip was performed on a GeneChip Hybridization oven 640, then dyed in the GeneChip Fluidics Station 450 and thereafter scanned with a GeneChip Scanner 3000. All of the equipment used was from the Affymetrix-Company (Affymetrix, High Wycombe, UK). All the necessary procedures needed for hybridization and scanning of chips were performed in the Affymetrix Core Facility of Medical Research Center, Medical Faculty Mannheim.

\section{RT-PCR}

cDNA synthesis was performed using SensiFAST cDNA Synthesis Kit from BIOLINE according to the manufacturer's instructions. The obtained cDNA was diluted 10 times and $1 \mu \mathrm{L}$ was used for RTPCR. Levels of mRNA from stabilin-1 and GDF-15, were quantified using TaqMan PCR primer mix (Eurofins, Germany) in the standard conditions. Primer sequences and probes are shown from the $5^{\prime}$ end to $3^{\prime}$ end direction.

For hsSTAB-1 the following sequence was used: FP: GCGACACCTTTTGTGAAC, RP: ATGCTTCTGCTTTCAG CC, Pr: FAM TTCGATGACTCACTGCTGGAGGAGGACTT.

For 18srRNA: FP: CCATTCGAACGTCTGCCCTAT, RP: TCACCCGTGGTCACCATG, Pr: ACTTTCGATGGTAG TCGCCGTGCCT.

Ready-to-use Taqman master mixes were used for GDF-15 (GDF-15, MIC-1, MIC1, NAG-1, PDF, PLAB, PTGF-B) Hs00171132_m1 (context sequence: CGCCAGAAGTGCG GCTGGGATCCGG) (Thermo Fisher Scientific).

Amplification was performed using Light cycler 480 systems (Roche Lifesciences). The expression levels of analyzed genes were normalized according to the 18 srRNA.

\section{Cytokine Secretion Assay}

The concentration of secreted GDF-15 was determined in macrophage culture supernatants using ELISA assays from R\&D systems (Wiesbaden, Germany) according to the manufacturer's instructions.

\section{Endocytosis Assay}

acLDL-Alexa488 (Invitrogen) was used as a ligand for endocytosis quantification. Endocytosis assays were performed in M0, M1 and M2, in general as described previously (22). Briefly, on day 6, acLDL-Alexa488 was added at a final concentration $2 \mu \mathrm{g} / \mathrm{mL}$ for flow cytometry quantification. For immunofluorescence analysis, macrophages were grown on coverslips, and acLDL-Alexa488 was added at a final concentration of $5 \mu \mathrm{g} / \mathrm{mL}$. Macrophages were incubated with the ligand for 30 minutes in the presence of 7,5\% $\mathrm{CO} 2$ at $37^{\circ} \mathrm{C}$. Cessation of endocytosis was achieved by placing cells on ice for flow cytometry. For immunofluorescence staining, cessation was achieved by immediate fixation with PFA as described (21), for M0, M1 and M2 treated with TiNPs, and for M1 non-treated with TiNPs. Since M0 and M2 without TiNPs were almost completely suspensional, sample preparation was performed using a Cytospin ${ }^{\mathrm{TM}} 4$ centrifuge (Thermo Fisher Scientific), and cytospins were fixed by PFA.

\section{Flow Cytometry}

Flow cytometry was used to quantify the uptake of the acLDLAlexa488. After endocytosis cessation, cells were harvested in ice-cold PBS. Fluorescent signal was quantified using BD FACS Canto II flow cytometer (FlowCore Mannheim, Germany). The data were analyzed using FlowJo 10.01 software.

\section{Immunofluorescence and Confocal Microscopy}

The following primary antibodies were used: anti-hstabilin-1 rabbit polyclonal serum RS1 (23) and mouse monoclonal antiEEA-1 (BD Biosciences). Secondary antibodies were Cy3conjugated donkey anti-rabbit IgG and Alexa647-conjugated donkey anti-mouse IgG (Dianova, Germany). In addition, all samples were stained with DAPI (Roche, Mannheim, Germany). Specificity of used antibodies was assessed in TiNPs treated cells with appropriate isotype control. Samples were mounted using DakoCytomation Fluorescent Mounting Medium (DakoCytomation, Hamburg, Germany). Confocal microscopy was performed using a Leica laser scanning spectral confocal microscope, model DM IRE2, equipped with an HCX PL Apo 63 $\times / 1.32$ numeric aperture oil objective (Leica Microsystems, Wetzlar, Germany). Excitation was done with an argon laser emitting at $488 \mathrm{~nm}$, a krypton laser emitting at $568 \mathrm{~nm}$, and a helium/neon laser emitting at $633 \mathrm{~nm}$. Images were acquired using a TCS SP8 DLS Leica inverted microscope and Leica Confocal software, version 2.5 (both from Leica Microsystems). Images were acquired using a sequential scan mode. For panel assembly, Adobe Photoshop version 6.0 (Adobe Systems, San Jose, CA) was used. Quantification of the fluorescence intensity for stabilin-1 and acLDL-Alexa488 was assessed using Qupath open source program. Three independent donors were analyzed and five different fields per donor were used for quantification. The program recognized individual cells and quantified the intensity of the signal for each cell. The means of fluorescent intensity were calculated for each field, for each donor, and, finally, for each condition: with or without TiNPs. The average fluorescent intensity of the overall cells was calculated.

\section{Statistics}

Statistical analysis was performed using GraphPad Prism 8 software (GraphPad Software Inc., USA). Bar graphs show mean \pm SEM. The significance of the data was analyzed using ratio paired Student's ttest. We considered a two-tailed $\mathrm{p}$-value of less than 0.05 to indicate statistical significance (confidence level 95\%). ns = non-significant, $\mathrm{p}<0.05, \mathrm{p} \leq 0.05^{\star *}, \mathrm{p} \leq 0.01{ }^{* * *} \mathrm{p} \leq 0.001$ and ${ }^{* * * *} \mathrm{p} \leq 0.001$.

\section{RESULTS}

\section{Microarray Analysis}

Microarray analysis revealed that TiNPs at a concentration of 100 ppm altered the expression of 5098 genes in M1 (IFN- $\gamma$ stimulated) and 4380 genes in M2 (IL-4-stimulated) macrophages. Additionally, 1980 genes were differentially regulated in both M1 and 
M2. The analysis revealed a significant downregulation of the multifunctional scavenger receptor stabilin-1 under TiNPs in M1 and M2 (fold change: $-3,12$ and -3,93, correspondingly). A contrary effect was seen for GDF-15, which was transcriptionally upregulated with TiNPs in both activation states (fold change: 2,94 and 2,54, correspondingly). Figure 1 shows the microarray analysis, in which every comparison was made between cells cultured with TiNPs versus cells without. The original array data for all differentially activated genes is available on the NCBI Gene Expression Omnibus (GEO) browser (Reference number GSE179543).

\section{TiNPs Stimulate GDF-15 and Suppress Stabilin-1 Gene Expression During Monocyte/Macrophage Differentiation}

To confirm the results of the microarray analysis, the expression of GDF-15 and stabilin-1 was verified by RT-PCR. The effect of TiNPs exposure on macrophages was examined after 6 days of monocyte differentiation into non-stimulated (M0), M1 and M2 macrophages. The expression of GDF-15 in monocytes (day 0) was minimal (Figure 2A). In all macrophage subtypes, the expression of GDF-15 was significantly increased when treated with TiNPs (Figure 2A). TiNPs upregulated the expression of GDF-15 in M0 by the average of 15 times $(p=0,0002)$ and in M1 by 10 times $(p<0,0001)$. Likewise, stimulation of M2 with TiNPs resulted in upregulation of GDF-15 expression by 6 times $(\mathrm{p}<0,0001)$. Additionally, GDF-15 expression was not significantly different between the different macrophage phenotypes, both with and without TiNPs. These results indicate that the exposure to TiNPs clearly has a stimulatory effect on the expression of GDF-15 in primary human monocytederived macrophages, and this expression is not influenced by macrophage differential activation.

In contrast, stabilin-1 expression was inhibited by TiNPs in all macrophage phenotypes ( $\mathrm{M} 0 \mathrm{p}=0,0008, \mathrm{M} 1 \mathrm{p}<0,0001$, and M2 p<0,0001). In monocytes (day 0) and M1, stabilin-1 mRNA levels were lower compared to M0 and M2 (Figure 2B).

\section{TiNPs Promote GDF-15 Secretion in Activated Macrophages}

The effect of TiNPs on GDF-15 secretion levels was assessed by ELISA in supernatants collected from macrophages cultured for 6 days (Figure 2C). In all macrophage subtypes, the secretion of GDF-15 was tremendously elevated after exposure to TiNPs. Upon the treatment with TiNPs, M0 increased GDF-15 secretion from almost 15 to approximately $380 \mathrm{pg} / \mathrm{mL}$ ( $\mathrm{p}=0,0105)$. M1 also showed increased GDF-15 secretion under TiNPs stimulation
A

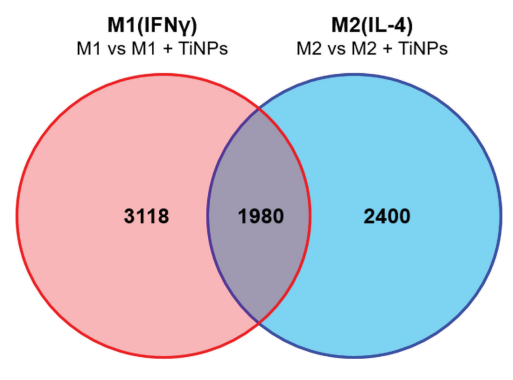

M1

C
B

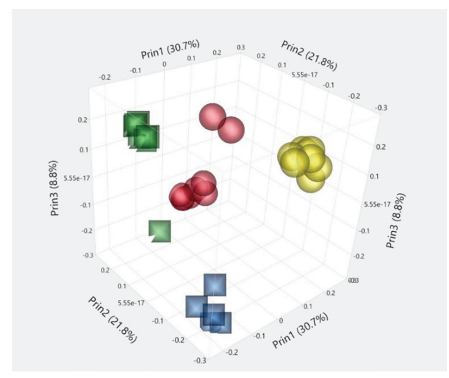

M2

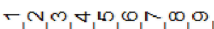

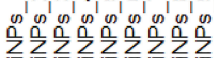
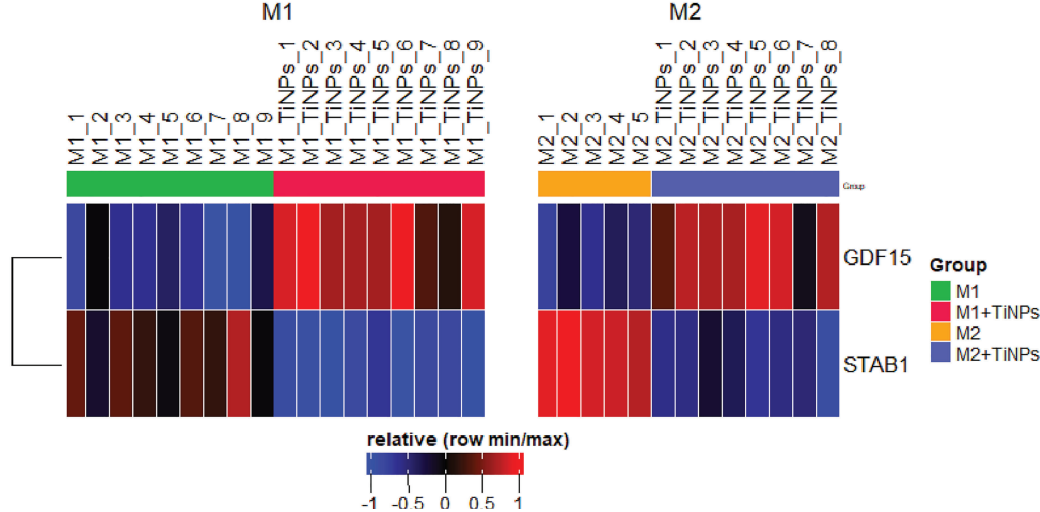

FIGURE 1 | Summary of microarray analysis of the effect of titanium nanoparticles on transcriptome of human inflammatory and healing macrophages. (A) Venn's diagram of differentially expressed genes in macrophages cultured in presence of TiNPs. Each number represents the number of genes differentially expressed in response to TiNPs. Each circle represents a population of macrophages with a different stimulation: Red - M1(IFN $\gamma$ ) and Blue - M2(IL-4). Intersecting points represent the number of genes differentially regulated by TiNPs in both stimulations conditions. (B) Clustering of the microarray data in a 3D scatterplot. Each sphere represents genes from one donor. Clustering of data from M1(IFN $\gamma$ ) is represented by yellow spheres - Control and red spheres - TiNPs); clustering of data from M2 (IL-4) is represented by blue squares - Control and green squares - TiNPs). (C) Heatmap of relative expression of stabilin-1 and GDF-15. Differential expression is shown between Control and TiNPs conditions M1 and M2, respectively. The number of donors differs for every condition depending on the availability of materials. 
A
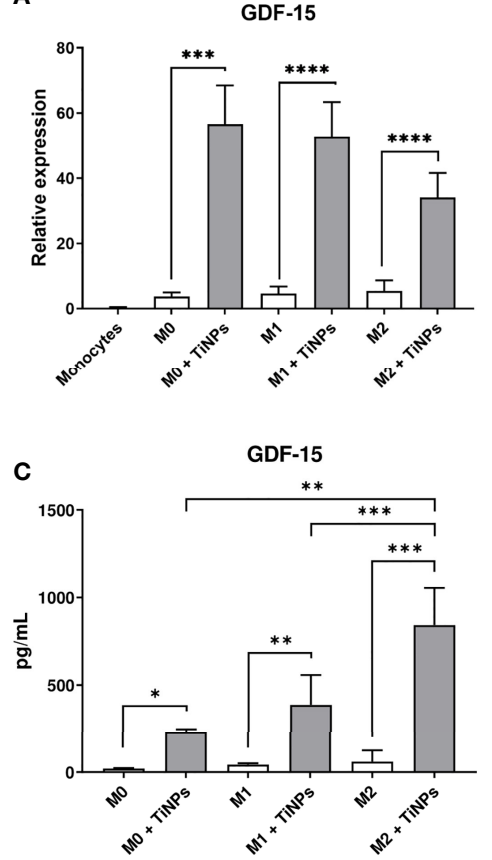

B

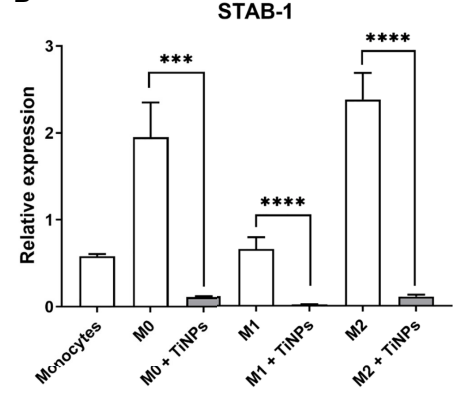

FIGURE 2 | Titanium nanoparticles have opposite effect on the expression of GDF-15 and stabilin-1 and strongly stimulate GDF-15 secretion in macrophages. mRNA levels were analyzed by RT-PCR in monocytes (day 0), and in non-stimulated (M0), M1 and M2 macrophages cultured for 6 days. (A) GDF-15 expression: $n$ = 3 for monocytes, $\mathrm{n}=7$ for M0, $\mathrm{n}=12$ for M1, and $\mathrm{n}=12$ for M2. (B) Stabilin-1 expression: $\mathrm{n}=4$ for monocytes, $\mathrm{n}=7$ for $\mathrm{M0}$, $\mathrm{n}=12$ for $\mathrm{M} 1$, and $\mathrm{n}=12$ for $\mathrm{M} 2$. (C) Supernatants of M0, M1 and M2 macrophages cultured for 6 days were analyzed by ELISA: $n=5$ for M0, $n=10$ for M1, $n=10$ for M2. Error bars indicate the means standard error of the results normalized to 18 SrRNA expression levels. ${ }^{*} p<0,05,{ }^{* \star} p<0,01,{ }^{\star \star *} p<0,001,{ }^{\star \star \star \star} p<0,0001$.

( $\mathrm{p}=0,0011)$. The highest GDF-15 secretion was observed in M2 under TiNPs stimulation (M0 vs M2 p=0,0086 and M1 vs M2 $\mathrm{p}=0,0095)$.

\section{TiNPs Decrease the Efficiency of Endocytosis}

We evaluated the effect of TiNPs on the uptake of fluorescently labeled acLDL, a known ligand of stabilin-1, in M0, M1 and M2 by flow cytometry. As depicted on the graph, the treatment with TiNPs decreased the efficiency of acLDL-Alexa488 endocytosis in all macrophage subtypes (Figure 3). After quantification, we found a significantly reduced endocytosis upon exposure to TiNPs compared to controls, which was consistent in all 7 analyzed individual donors. acLDL endocytosis was higher in M0 ( $p=0,0033)$ and M2 $(p=0,0004)$ controls as compared to M1 control (Figure 3). This data indicates that acLDL endocytosis is negatively affected by TiNPs in all macrophage phenotypes.

\section{TiNPs Disrupt Stabilin-1-Mediated Endocytic Trafficking}

To further explore the effect of TiNPs on stabilin-1-mediated internalization and intracellular transport along the endocytic pathway, confocal microscopy was used to visualize the localization of stabilin-1 and acLDL-Alexa488. Since M2 expresses maximal levels of stabilin-1 and have enhanced endocytic properties compared to M0 and M1, M2 subtype was used for the immunofluorescence analysis (24). EEA1-positive early/sorting endosomes is a major vesicular compartment, where

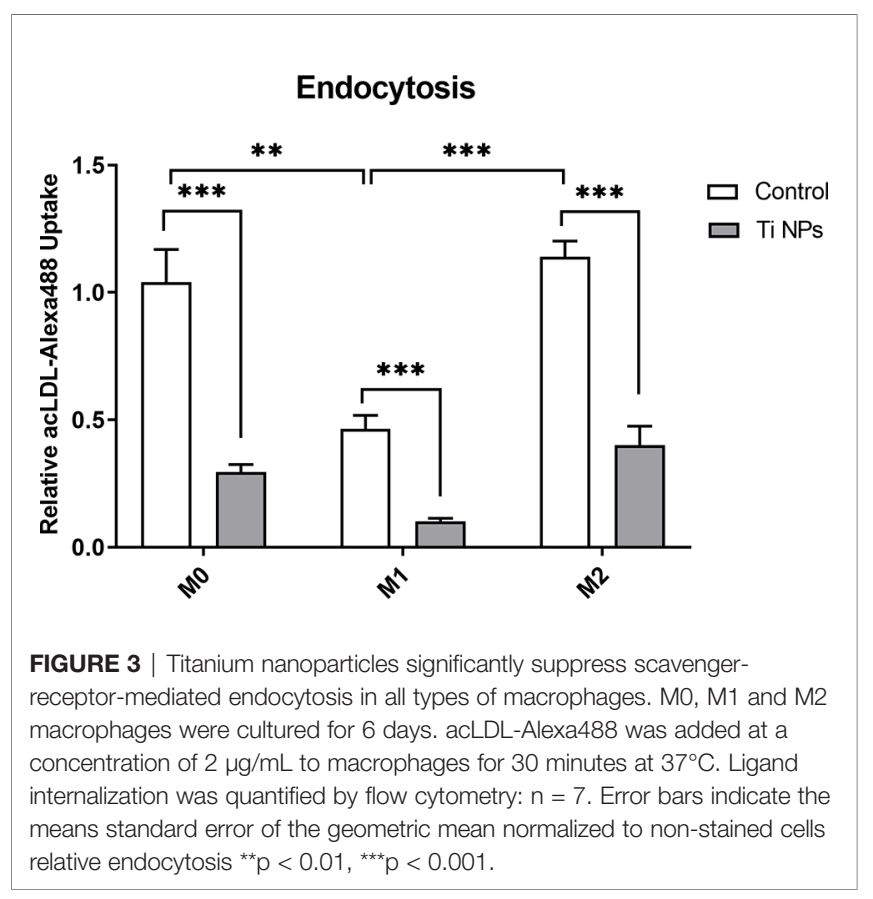


stabilin-1 is localized in M2 (21). Therefore, we assessed its intracellular localization using anti-EEA1 and anti-stabilin-1 rabbit polyclonal RS1 antibody. In the absence of TiNPs, M2 expressed high levels of stabilin-1. Additionally, the majority of acLDL-Alexa488 was co-localized with stabilin-1, and was efficiently delivered to EEA1-positive early/sorting endosomes, while EEA1 marked irregularly shaped large endosomes (Figure 4A). Treatment with TiNPs resulted in the disruption of endosomal compartment. Only a small amount of remaining EEA1 endosomes was detected, and internalization of acLDL-Alexa488 was almost abrogated (Figure 4B). After TiNPs treatment, stabilin1 was expressed only in a small percentage of $\mathrm{M} 2$, and only in stabilin-1+cells internalization of acLDL-Alexa488 was still detectable. Moreover, the mean fluorescence intensity of both acLDL-488 and stabilin-1 was significantly lower in M2 treated with TiNPs ( $p=0,008$ and $p=0,0118$, respectively) (Figures 4C, D). Overall, the immunofluorescence analysis confirmed the suppressive effect of TiNPs on stabilin-1 expression on protein level and stabilin-1-mediated endocytosis.

\section{DISCUSSION}

Here, the effect of TiNPs (debris of titanium implants) on transcriptome of human healing macrophages was analyzed for
A
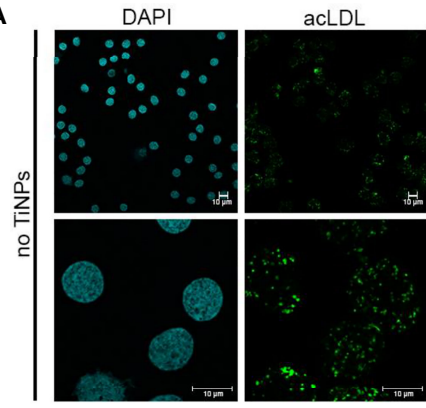

B
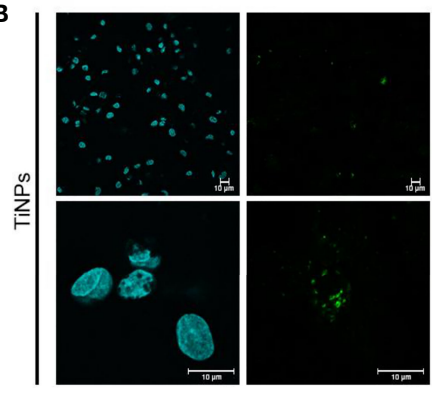

C

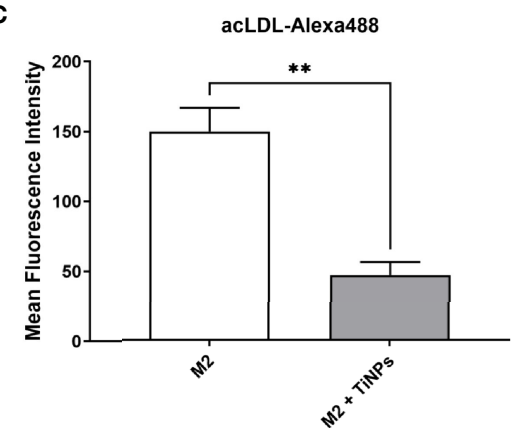

Stab-1
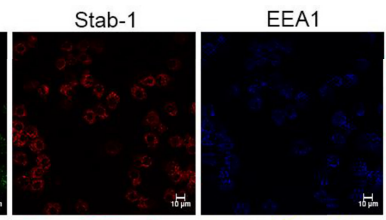

Merged
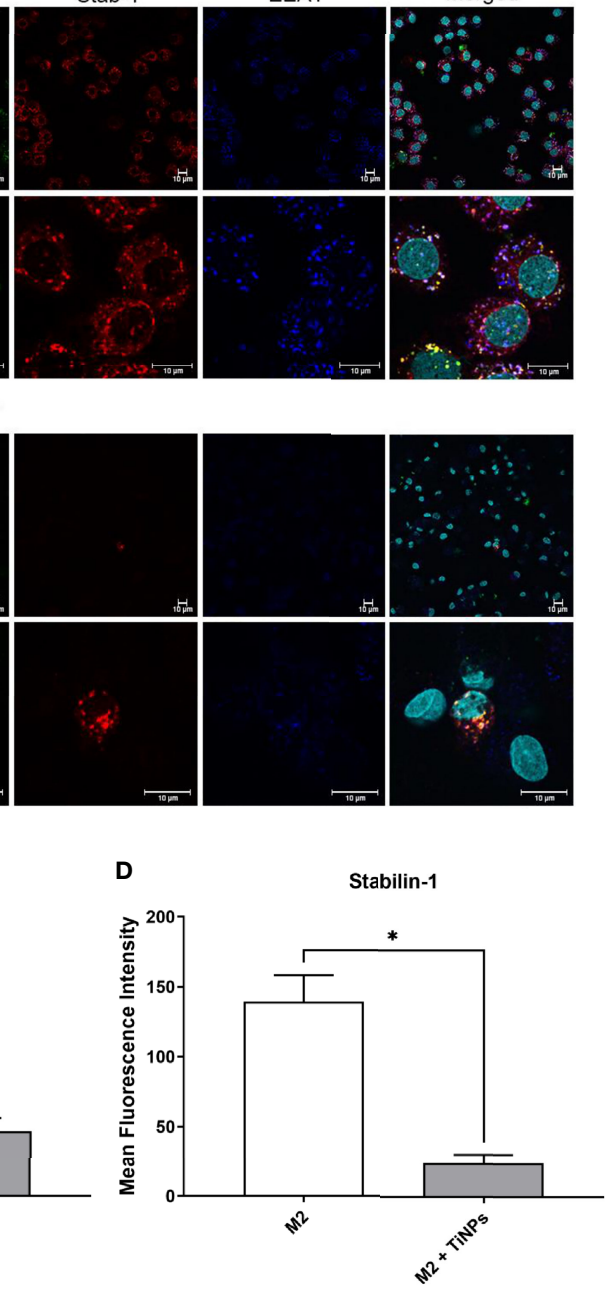

FIGURE 4 | Confocal microscopy analysis of the suppressive TiNP effect on the stabilin-1-mediated endocytosis in healing M2 macrophages. M2 macrophages were exposed to stabilin-1 ligand acLDL-Alexa488 at a concentration of $5 \mu \mathrm{g} / \mathrm{mL}$ for 30 minutes. M2 derived from monocytes of 3 individual donors were used for each type of analysis. (A) Representative images of confocal microscopy analysis of M2 in absence of TiNPs. (B) Representative images of confocal microscopy analysis of M2 in presence of TiNPs. Visualization of nuclei was performed using DAPI (visualized in cyan). acLDL-Alexa488 is shown in green. Stabilin-1 was detected by RS1 rabbit polyclonal antibody and Cy3-conjugated anti-rabbit secondary antibody (visualized in red). EEA1 was detected using anti-EEA1 mouse antibody and Alexa-647-conjugated secondary antibody (visualized in blue). Representative images of single cells are shown for M2. Scale bars: 10 um. (C) Quantification of confocal microscopy images for the internalized acLDL-Alexa488 and (D) quantification of confocal microscopy images for expression of stabilin-1. For every 3 individual donors, 5 different fields of images were assessed using Qupath open source program. The mean fluorescence intensity was calculated by averaging the intensity of each cell per condition. Error bars indicate the means standard error of the results. ${ }^{\star} \mathrm{p}<0.05$, ${ }^{\star \star} \mathrm{p}<0.01$. 
the first time. We found that TiNPs suppress essential for healing scavenging function of macrophages, mediated by stabilin-1. At the same time, we found that TiNPs strongly upregulate the production of GDF-15.

GDF-15 is a multifunctional cytokine and remote member from the glial cell-derived neurotrophic factor (GDNF) family and TGF- $\beta$ superfamily $(25,26)$. Its membrane receptor, GDNF family receptor $\alpha$-like (GFRAL), was considered to be uniquely expressed in the hindbrain $(25,27,28)$. Recent data showed that GFRAL expression is also present in human adipocytes and prostate cancer cells $(29,30)$. GFRAL was also reported to be expressed in endothelial cells and to mediate GDF-15-induced pro-angiogenic effect (31).

GDF-15 has been recognized as a potential diagnostic and prognostic biomarker for several diseases, including cancer, cardiovascular disease, sepsis, and recently, $\operatorname{COVID-19}(32,33)$. GDF-15 is known for being a pleiotropic cytokine mainly expressed and secreted during stress conditions and inflammation, and its function seems to be protective (34-37). Recently, Cimino et al. discovered that GDF-15 induces a rise in glucocorticoid levels through GFRAL signaling upon endoplasmic reticulum (ER) stress induction in mice, which undercovers GDF-15 centrally mediated-anti-inflammatory response (38). Its role in immune tolerance was also addressed by Luan et al, who discovered that GDF-15 rise during bacterial inflammation increases hepatic triglyceride production, mediating cardiac protection and promoting survival (36). Other studies have shown detrimental consequences of increased GDF-15, highlighting a contextdependent action (39). GDF-15 has been correlated with fibrotic diseases. For instance, Govaere et al. quantified GDF-15 expression and secretion levels in hepatic tissue using transcriptomic and proteomic analysis and found that GDF-15 positively correlates to fibrosis progression in nonalcoholic fatty liver disease (NAFLD) (40). Nevertheless, its complete molecular mechanism and signaling pathway have not been fully established.

In macrophages, GDF-15 expression is increased under the effect of IL-4, IL-1 $\beta$, TNF- $\alpha$, IL-2 and M-CSF (41-43). Previous studies have found elevated GDF-15 expression in M1 (but not M2) macrophages and alveolar macrophages upon acute exposure to TiNPs and silica microspheres ( 4 hours and 16 hours, respectively) $(19,44)$. Our findings complement this information by pointing out a persistent effect of TiNPs (6 days) on GDF-15 gene expression in all macrophage phenotypes. Furthermore, GDF-15 was preferentially secreted by M2 macrophages under TiNPs treatment.

It is worth mentioning that the TiNPs-induced proinflammatory reaction has been associated with increased production of reactive oxygen species (ROS) and mitochondrial damage (45). Interestingly, GDF-15 production is induced by mitochondrial uncoupling and by the treatment with mitochondrial inhibitors, including metformin $(46,47)$. Likewise, TiNPs induce ER stress and disrupt the mitochondrial-associated ER membranes (48). Therefore, identified by us increased GDF-15 expression could be explained by the mitochondrial damage generated by TiNPs. Another documented effect of TiNPs on macrophages is lysosomal damage. Particularly, the here used rutile TiNPs, have a detrimental impact on lysosomal permeability (49). Recently, Kim et al. found that transcription factor EB (TFEB), a regulator of energy expenditure and autophagy inductor, binds to GDF-15 promotor and induced its expression after lysosomal stress induction in macrophages (50). The potential lysosomal damage caused by exposure to TiNPs could also explain the rise in GDF-15 levels. This hypothesis is consistent with the observation from our transcriptome analysis, showing a significant upregulation of TFEB expression (M1: fold change $=0,43, \mathrm{p}$ value $=0,03 ; \mathrm{M} 2$ : fold change $=1,55, \mathrm{p}$-value $<0,0001)$ in both human $\mathrm{CD}_{14}{ }^{+}$derived M1 and M2 after 6 days of TiNPs exposure in culture (Reference number GSE179543).

Stabilin-1 is an established biomarker in M2 macrophages essential for their clearance function in health and pathology (51). Multifunctional scavenger receptor stabilin-1 (STAB-1, FEEL-1, CLEVER-1, KIAA0246) is a transmembrane scavenger and sorting receptor expressed on tissue macrophages and noncontinuous endothelial cells $(21,24)$. In human monocytederived macrophages, the expression of stabilin- 1 is induced by stimulation with IL-4 and dexamethasone (23). Stabilin-1 controls the balance between inflammation and tissue remodeling by scavenging and targeting for secretion multiple extracellular regulatory proteins including SPARC, SI-CLP, YKL-39 and placental lactogen $(22,52-54)$. It also mediates the clearance of apoptotic bodies as well as modified lipoproteins $(55,56)$. Here, we found that stabilin-1 expression is highly suppressed under TiNPs treatment in all activation states. Stabilin-1 decreased expression supports the previous observations that TiNPs promote a shift to M1 polarization and suppressed M2 response (19). Our flow cytometry data showed a markedly decreased endocytosis efficiency in all activation states after co-culturing with TiNPs. The fold change was higher for $\mathrm{M} 0$ and $\mathrm{M} 2$, which are reported to mediate more actively endocytosis than M1 (57). This is also consistent with our observation that M0 and M2 expressed more stabilin-1 than M1. Quantitative confocal microscopy analysis further confirmed the abrogation of stabilin-1 expression in M2 co-cultured with TiNPs. A decrease in stabilin-1 can also result in impaired clearance of SPARC, modified lipoproteins and apoptotic bodies, the accumulation of which is frequent in chronic inflammation and fibrosis $(53,55,56,58)$. Moreover, deficiency of stabilin-1 in mice aggravates inflammation by increased inflammatory macrophage activation and enhanced IgM production (59).

We have previously identified that GDF-15 is an endocytic ligand of stabilin-1 (60). We showed that impaired clearance of GDF-15 in STAB-1-/-STAB-2-/- mice leads to severe glomerular fibrosis and mild perisinusoidal hepatic fibrosis. With this context, the potential implications of increased secretion of GDF-15 added to dysfunction of its clearance receptor in implant holders are numerous. The remark that all macrophage phenotypes highly secreted GDF-15 under TiNPs, highlights the possibility that GDF-15 acts in an autocrine and paracrine matter. For instance, Jung et al. showed that rGDF-15 treatment decreases the expression of IL-6, nitric oxide synthase 2 (NOS2) and TNF- $\alpha$ and promotes an M2 polarization by augmenting Arg-1, Retnla and chitinase 3-like 3 protein (Ym1) 
production in murine blood marrow-derived macrophages (42). Therefore, GDF-15 could promote a change of phenotype from M1 to M2 in resident and recruited macrophages. Possible receptors for this action are TGF- $\beta$ RI and II, which were identified to bind to GDF-15 on dendritic cells promoting immune tolerance (61). An accumulation of GDF-15 in the peri-implant tissues could also impact other surrounding cells. Because of its pro-angiogenic properties, increased GDF-15 in the tissue could promote microvasculature proliferation via GFRAL (31). Other possible target cells of macrophageproduced GDF-15 are osteoblasts and osteoclasts precursors. Wakchoure et al, for instance, observed that GDF-15 promotes osteolytic lesions in mice models of prostate cancer with bone metastasis (62). Hinoi et al. showed that anti-GDF-15 decreases bone loss and inhibits osteoclastogenesis in mice models (63). Westhrin et al. showed that GDF-15 promotes osteoclast activation in osteoclasts derived from human peripheral blood mononuclear cells while decreasing osteoblast differentiation (64). Contrastingly, Vanhara et al, showed that GDF-15 inhibited the formation of mature osteoclasts in RAW264.7 cells (65). Interestingly, GDF-15 secretion varies with titanium implant surface modifications, being particularly promoted by rough surfaces $(66,67)$. However, the real impact of GDF-15 on osteogenesis and implant osseointegration is still to be clarified.

Titanium debris have been shown to induce M1-polarization and to significantly increase the production of pro-inflammatory cytokines. IL-1 $\beta$, IL- 6 and TNF- $\alpha$, which expression and secretion is upregulated in macrophages after exposure to titanium debris, also seem to affect the degree of bone resorption in paracrine matter. Eger et al. demonstrated that the blockade of IL-1 $\beta$, IL- 6 and TNF$\alpha$, using neutralizing antibodies, prevents osteolysis due to titanium particles in mouse models (68). These findings highlights the importance of macrophage system on the implant success and opens the door to explore new targetable mechanisms to prevent implant failure, such as stabilin-1/GDF-15.

Titanium implants located in other organs, such as in the heart, can also promote fibrotic reactions via increased GDF-15 and decreased stabilin-1 expression. Indeed, GDF-15 has been previously associated with heart remodeling and heart failure (69). Implants containing titanium are frequently used in cardiology, for instance, in patients with reduced ventricular function (70). A release of TiNPs and, consequently, an uncontrolled increase in GDF-15 could in long term promote cardiac fibrosis and further exacerbate heart failure.

\section{REFERENCES}

1. Dux KE. Implantable Materials Update. Clin Podiatr Med Surg (2019) 36 (4):535-42. doi: 10.1016/j.cpm.2019.06.001

2. Elani HW, Starr JR, Da Silva JD, Gallucci GO. Trends in Dental Implant Use in the U.S., 1999-2016, and Projections to 2026. J Dent Res (2018) 97 (13):1424-30. doi: 10.1177/00220345187925672

3. He J, Chen G, Liu M, Xu Z, Chen H, Yang L, et al. Scaffold Strategies for Modulating Immune Microenvironment During Bone Regeneration. Mater Sci Eng C Mater Biol Appl (2020) 108:110411. doi: 10.1016/j.msec.2019.110411

4. Kzhyshkowska J, Gudima A, Riabov V, Dollinger C, Lavalle P, Vrana NE. Macrophage Responses to Implants: Prospects for Personalized
Myelocytic depletion after clodronate treatment significantly reduces serum GDF-15 in mice, highlighting macrophages as a significant source of systemic GDF-15 (50). Therefore, it is feasible that macrophage secreted GDF-15 translates into a significant increase of GDF-15 circulating levels, increasing glucocorticoid levels due to TiNPs-induced ER stress, which could systemically lessen the pro-inflammatory effect of TiNPs $(38,48)$.

In summary, the dual effect of TiNPs on the GDF-15 overproduction and strong suppression of stabilin-1 clearance function in macrophages can be a detrimental effect of titanium implant debris formed with the time, which interferes with the long-term healthy implant integration, damages the balance between inflammation and healing processes, and is detrimental for patients. GDF-15 can potentially have also systemic effects and can be considered for therapeutic targeting to eliminate titanium implant-induced complications.

\section{DATA AVAILABILITY STATEMENT}

The datasets presented in this study can be found in online repositories. The names of the repository/repositories and accession number(s) can be found below: https://www.ncbi. nlm.nih.gov/, GSE179543.

\section{AUTHOR CONTRIBUTIONS}

JK, LS-B, and TS designed the project. LS-B, TS, LS, CT, and CS performed experiments. LS-B, TS, LS, CT, and JK have analysed data. LS-B, JK, TS, and HK wrote the manuscript. All authors contributed to the article and approved the submitted version.

\section{FUNDING}

The work was performed with the financial support of ERA-NET RUS Plus CoatDegraBac project (for JK).

\section{ACKNOWLEDGMENTS}

We gratefully acknowledge the excellent technical support of Stefanie Uhlig, the FlowCore Mannheim Medical Faculty.

Medicine. J Leukoc Biol (2015) 98(6):953-62. doi: 10.1189/ jlb.5VMR0415-166R

5. Orecchioni M, Ghosheh Y, Pramod AB, Ley K. Macrophage Polarization: Different Gene Signatures in M1(LPS+) vs. Classically and M2(LPS-) vs. Alternatively Activated Macrophages. Front Immunol (2019) 10:1084. doi: 10.3389/fimmu.2019.01084

6. Bellora F, Castriconi R, Doni A, Cantoni C, Moretta L, Mantovani A, et al. MCSF Induces the Expression of a Membrane-Bound Form of IL-18 in a Subset of Human Monocytes Differentiating In Vitro Toward Macrophages. Eur J Immunol (2012) 42(6):1618-26. doi: 10.1002/eji.201142173

7. Amengual-Penafiel L, Cordova LA, Constanza Jara-Sepulveda M, BranesAroca M, Marchesani-Carrasco F, Cartes-Velasquez R. Osteoimmunology 
Drives Dental Implant Osseointegration: A New Paradigm for Implant Dentistry. Jpn Dent Sci Rev (2021) 57:12-9. doi: 10.1016/j.jdsr.2021.01.001

8. Jonas K, Nils W, Alexander D, Stefan B, Henning W, Thilo F. The Etiology of Revision Total Hip Arthroplasty: Current Trends in a Retrospective Survey of 3450 Cases. Arch Orthop Trauma Surg (2020) 140(9):1265-73. doi: 10.1007/ s00402-020-03514-3

9. Kulshrestha V, Datta B, Mittal G, Kumar S. Epidemiology of Revision Total Knee Arthroplasty: A Single Center's Experience. Indian J Orthop (2019) 53 (2):282-8. doi: 10.4103/ortho.IJOrtho_127_17

10. Vanhegan IS, Malik AK, Jayakumar P, Ul Islam S, Haddad FS. A Financial Analysis of Revision Hip Arthroplasty: The Economic Burden in Relation to the National Tariff. J Bone Joint Surg Br (2012) 94(5):619-23. doi: 10.1302/ 0301-620X.94B5.27073

11. Matusiewicz H. Potential Release of In Vivo Trace Metals From Metallic Medical Implants in the Human Body: From Ions to Nanoparticles-a Systematic Analytical Review. Acta Biomater (2014) 10(6):2379-403. doi: 10.1016/j.actbio.2014.02.027

12. Noronha Oliveira M, Schunemann WVH, Mathew MT, Henriques B, Magini RS, Teughels W, et al. Can Degradation Products Released From Dental Implants Affect Peri-Implant Tissues? J Periodontal Res (2018) 53(1):1-11. doi: $10.1111 /$ jre.12479

13. Curtin JP, Wang M. Are Clinical Findings of Systemic Titanium Dispersion Following Implantation Explained by Available In Vitro Evidence? An Evidence-Based Analysis. J Biol Inorg Chem (2017) 22(6):799-806. doi: 10.1007/s00775-017-1464-1

14. Alinovi R, Goldoni M, Pinelli S, Campanini M, Aliatis I, Bersani D, et al. Oxidative and Pro-Inflammatory Effects of Cobalt and Titanium Oxide Nanoparticles on Aortic and Venous Endothelial Cells. Toxicol In Vitro (2015) 29(3):426-37. doi: 10.1016/j.tiv.2014.12.007

15. Han SG, Newsome B, Hennig B. Titanium Dioxide Nanoparticles Increase Inflammatory Responses in Vascular Endothelial Cells. Toxicology (2013) 306:1-8. doi: 10.1016/j.tox.2013.01.014

16. Hong F, Wu N, Ge Y, Zhou Y, Shen T, Qiang Q, et al. Nanosized Titanium Dioxide Resulted in the Activation of TGF-Beta/Smads/p38MAPK Pathway in Renal Inflammation and Fibration of Mice. J BioMed Mater Res A (2016) 104(6):1452-61. doi: 10.1002/jbm.a.35678

17. Kheder W, Soumya S, Samsudin AR. Impact of Titanium Dioxide Particle Size on Macrophage Production of Intracellular Reactive Oxygen Species. Arch Oral Biol (2021) 127:105133. doi: 10.1016/j.archoralbio.2021.105133

18. Fort BP, Dubyak GR, Greenfield EM. Lysosomal Disruption by Orthopedic Wear Particles Induces Activation of the NLRP3 Inflammasome and Macrophage Cell Death by Distinct Mechanisms. J Orthop Res (2021) 39 (3):493-505. doi: 10.1002/jor.24826

19. Pajarinen J, Kouri VP, Jamsen E, Li TF, Mandelin J, Konttinen YT. The Response of Macrophages to Titanium Particles is Determined by Macrophage Polarization. Acta Biomater (2013) 9(11):9229-40. doi: 10.1016/j.actbio.2013.06.027

20. Wischhusen J, Melero I, Fridman WH. Growth/Differentiation Factor-15 (GDF-15): From Biomarker to Novel Targetable Immune Checkpoint. Front Immunol (2020) 11:951. doi: 10.3389/fimmu.2020.00951

21. Kzhyshkowska J, Gratchev A, Martens JH, Pervushina O, Mamidi S, Johansson S, et al. Stabilin-1 Localizes to Endosomes and the Trans-Golgi Network in Human Macrophages and Interacts With GGA Adaptors. J Leukoc Biol (2004) 76(6):1151-61. doi: 10.1189/jlb.0504300

22. Kzhyshkowska J, Gratchev A, Schmuttermaier C, Brundiers H, Krusell L, Mamidi S, et al. Alternatively Activated Macrophages Regulate Extracellular Levels of the Hormone Placental Lactogen via Receptor-Mediated Uptake and Transcytosis. J Immunol (2008) 180(5):3028-37. doi: 10.4049/ jimmunol.180.5.3028

23. Politz O, Gratchev A, McCourt PA, Schledzewski K, Guillot P, Johansson S, et al. Stabilin-1 and -2 Constitute a Novel Family of Fasciclin-Like Hyaluronan Receptor Homologues. Biochem J (2002) 362(Pt 1):155-64. doi: $10.1042 /$ bj3620155

24. Kzhyshkowska J, Gratchev A, Goerdt S. Stabilin-1, a Homeostatic Scavenger Receptor With Multiple Functions. J Cell Mol Med (2006) 10(3):635-49. doi: 10.1111/j.1582-4934.2006.tb00425.x

25. Hsu JY, Crawley S, Chen M, Ayupova DA, Lindhout DA, Higbee J, et al. NonHomeostatic Body Weight Regulation Through a Brainstem-Restricted
Receptor for GDF15. Nature (2017) 550(7675):255-9. doi: 10.1038/ nature 24042

26. Strelau J, Bottner M, Lingor P, Suter-Crazzolara C, Galter D, Jaszai J, et al. GDF-15/MIC-1 a Novel Member of the TGF-Beta Superfamily. J Neural Transm Suppl (2000) 60):273-6 doi: 10.1007/978-3-7091-6301-6_18

27. Emmerson PJ, Wang F, Du Y, Liu Q, Pickard RT, Gonciarz MD, et al. The Metabolic Effects of GDF15 are Mediated by the Orphan Receptor GFRAL. Nat Med (2017) 23(10):1215-9. doi: 10.1038/nm.4393

28. Mullican SE, Lin-Schmidt X, Chin CN, Chavez JA, Furman JL, Armstrong AA, et al. GFRAL is the Receptor for GDF15 and the Ligand Promotes Weight Loss in Mice and Nonhuman Primates. Nat Med (2017) 23(10):1150-7. doi: 10.1038/nm.4392

29. Huang M, Narita S, Koizumi A, Nara T, Numakura K, Satoh S, et al. Macrophage Inhibitory Cytokine-1 Induced by a High-Fat Diet Promotes Prostate Cancer Progression by Stimulating Tumor-Promoting Cytokine Production From Tumor Stromal Cells. Cancer Commun (Lond) (2021) 41 (5):389-403. doi: 10.1002/cac2.12137

30. Laurens C, Parmar A, Murphy E, Carper D, Lair B, Maes P, et al. Growth and Differentiation Factor 15 is Secreted by Skeletal Muscle During Exercise and Promotes Lipolysis in Humans. JCI Insight (2020) 5(6):1-14. doi: 10.1172/ jci.insight. 131870

31. Lee J, Jin YJ, Lee MS, Kim YM, Lee H. Macrophage Inhibitory Cytokine-1 Promotes Angiogenesis by Eliciting the GFRAL-Mediated Endothelial Cell Signaling. J Cell Physiol (2021) 236(5):4008-23. doi: 10.1002/jcp.30144

32. Lockhart SM, Saudek V, O'Rahilly S. GDF15: A Hormone Conveying Somatic Distress to the Brain. Endocr Rev (2020) 41(4):610-42. doi: 10.1210/endrev/ bnaa007

33. Teng X, Zhang J, Shi Y, Liu Y, Yang Y, He J, et al. Comprehensive Profiling of Inflammatory Factors Revealed That Growth Differentiation Factor-15 Is an Indicator of Disease Severity in COVID-19 Patients. Front Immunol (2021) 12:662465. doi: 10.3389/fimmu.2021.662465

34. Johnen H, Kuffner T, Brown DA, Wu BJ, Stocker R, Breit SN. Increased Expression of the TGF-B Superfamily Cytokine MIC-1/GDF15 Protects ApoE $(-/-)$ Mice From the Development of Atherosclerosis. Cardiovasc Pathol (2012) 21(6):499-505. doi: 10.1016/j.carpath.2012.02.003

35. Kim KH, Kim SH, Han DH, Jo YS, Lee YH, Lee MS. Growth Differentiation Factor 15 Ameliorates Nonalcoholic Steatohepatitis and Related Metabolic Disorders in Mice. Sci Rep (2018) 8(1):6789. doi: 10.1038/s41598-018-25098-0

36. Luan HH, Wang A, Hilliard BK, Carvalho F, Rosen CE, Ahasic AM, et al. GDF15 Is an Inflammation-Induced Central Mediator of Tissue Tolerance. Cell (2019) 178(5):1231-44.e11. doi: 10.1016/j.cell.2019.07.033

37. Conte M, Martucci M, Mosconi G, Chiariello A, Cappuccilli M, Totti V, et al. GDF15 Plasma Level Is Inversely Associated With Level of Physical Activity and Correlates With Markers of Inflammation and Muscle Weakness. Front Immunol (2020) 11:915. doi: 10.3389/fimmu.2020.00915

38. Cimino I, Kim H, Tung YCL, Pedersen K, Rimmington D, Tadross JA, et al. Activation of the Hypothalamic-Pituitary-Adrenal Axis by Exogenous and Endogenous GDF15. Proc Natl Acad Sci USA (2021) 118(27):1-10. doi: 10.1073/pnas. 2106868118

39. Santos I, Colaco HG, Neves-Costa A, Seixas E, Velho TR, Pedroso D, et al. CXCL5-Mediated Recruitment of Neutrophils Into the Peritoneal Cavity of Gdf15-Deficient Mice Protects Against Abdominal Sepsis. Proc Natl Acad Sci USA (2020) 117(22):12281-7. doi: 10.1073/pnas.1918508117

40. Govaere O, Cockell S, Tiniakos D, Queen R, Younes R, Vacca M, et al. Transcriptomic Profiling Across the Nonalcoholic Fatty Liver Disease Spectrum Reveals Gene Signatures for Steatohepatitis and Fibrosis. Sci Transl Med (2020) 12(572):1-17. doi: 10.1126/scitranslmed.aba4448

41. Bootcov MR, Bauskin AR, Valenzuela SM, Moore AG, Bansal M, He XY, et al. MIC-1, a Novel Macrophage Inhibitory Cytokine, is a Divergent Member of the TGF-Beta Superfamily. Proc Natl Acad Sci USA (1997) 94(21):11514-9. doi: $10.1073 /$ pnas.94.21.11514

42. Jung SB, Choi MJ, Ryu D, Yi HS, Lee SE, Chang JY, et al. Reduced Oxidative Capacity in Macrophages Results in Systemic Insulin Resistance. Nat Commun (2018) 9(1):1551. doi: 10.1038/s41467-018-03998-z

43. Ratnam NM, Peterson JM, Talbert EE, Ladner KJ, Rajasekera PV, Schmidt CR, et al. NF-kappaB Regulates GDF-15 to Suppress Macrophage Surveillance During Early Tumor Development. J Clin Invest (2017) 127(10):3796-809. doi: 10.1172/JCI91561 
44. Kersting M, Olejnik M, Rosenkranz N, Loza K, Breisch M, Rostek A, et al. Subtoxic Cell Responses to Silica Particles With Different Size and Shape. Sci Rep (2020) 10(1):21591. doi: 10.1038/s41598-020-78550-5

45. Hu Q, Zhao F, Fan M, He C, Yang X, Huang Z, et al. The Influence of Titanium Dioxide Nanoparticles on Their Cellular Response to Macrophage Cells. Comp Biochem Physiol C Toxicol Pharmacol (2019) 223:42-52. doi: 10.1016/j.cbpc.2019.05.006

46. Ost M, Igual Gil C, Coleman V, Keipert S, Efstathiou S, Vidic V, et al. MuscleDerived GDF15 Drives Diurnal Anorexia and Systemic Metabolic Remodeling During Mitochondrial Stress. EMBO Rep (2020) 21(3):e48804. doi: 10.15252/ embr.201948804

47. Yang M, Darwish T, Larraufie P, Rimmington D, Cimino I, Goldspink DA, et al. Inhibition of Mitochondrial Function by Metformin Increases Glucose Uptake, Glycolysis and GDF-15 Release From Intestinal Cells. Sci Rep (2021) 11(1):2529. doi: 10.1038/s41598-021-81349-7

48. Yu KN, Chang SH, Park SJ, Lim J, Lee J, Yoon TJ, et al. Titanium Dioxide Nanoparticles Induce Endoplasmic Reticulum Stress-Mediated Autophagic Cell Death via Mitochondria-Associated Endoplasmic Reticulum Membrane Disruption in Normal Lung Cells. PloS One (2015) 10(6):e0131208. doi: 10.1371/journal.pone. 0131208

49. Yu Q, Wang H, Peng Q, Li Y, Liu Z, Li M. Different Toxicity of Anatase and Rutile TiO2 Nanoparticles on Macrophages: Involvement of Difference in Affinity to Proteins and Phospholipids. J Hazard Mater (2017) 335:125-34. doi: 10.1016/j.jhazmat.2017.04.026

50. Kim J, Kim SH, Kang H, Lee S, Park SY, Cho Y, et al. TFEB-GDF15 Axis Protects Against Obesity and Insulin Resistance as a Lysosomal Stress Response. Nat Metab (2021) 3(3):410-27. doi: 10.1038/s42255-021-00368-w

51. Kzhyshkowska J. Multifunctional Receptor Stabilin-1 in Homeostasis and Disease. ScientificWorldJournal (2010) 10:2039-53. doi: 10.1100/tsw.2010.189

52. Kzhyshkowska J, Mamidi S, Gratchev A, Kremmer E, Schmuttermaier C, Krusell L, et al. Novel Stabilin-1 Interacting Chitinase-Like Protein (SI-CLP) is Up-Regulated in Alternatively Activated Macrophages and Secreted via Lysosomal Pathway. Blood (2006) 107(8):3221-8. doi: 10.1182/blood-200507-2843

53. Kzhyshkowska J, Workman G, Cardo-Vila M, Arap W, Pasqualini R, Gratchev A, et al. Novel Function of Alternatively Activated Macrophages: Stabilin-1-Mediated Clearance of SPARC. J Immunol (2006) 176(10):582532. doi: 10.4049/jimmunol.176.10.5825

54. Liu T, Larionova I, Litviakov N, Riabov V, Zavyalova M, Tsyganov M, et al. Tumor-Associated Macrophages in Human Breast Cancer Produce New Monocyte Attracting and Pro-Angiogenic Factor YKL-39 Indicative for Increased Metastasis After Neoadjuvant Chemotherapy. Oncoimmunology (2018) 7(6):e1436922. doi: 10.1080/2162402X.2018.1436922

55. Kzhyshkowska J, Gratchev A, Brundiers H, Mamidi S, Krusell L, Goerdt S. Phosphatidylinositide 3-Kinase Activity is Required for Stabilin-1-Mediated Endosomal Transport of acLDL. Immunobiology (2005) 210(2-4):161-73. doi: 10.1016/j.imbio.2005.05.022

56. Park SY, Jung MY, Lee SJ, Kang KB, Gratchev A, Riabov V, et al. Stabilin-1 Mediates Phosphatidylserine-Dependent Clearance of Cell Corpses in Alternatively Activated Macrophages. J Cell Sci (2009) 122(Pt 18):3365-73. doi: $10.1242 /$ jcs. 049569

57. Tarique AA, Logan J, Thomas E, Holt PG, Sly PD, Fantino E. Phenotypic, Functional, and Plasticity Features of Classical and Alternatively Activated Human Macrophages. Am J Respir Cell Mol Biol (2015) 53(5):676-88. doi: 10.1165/rcmb.2015-0012OC

58. Riley HJ, Kelly RR, Van Laer AO, Neff LS, Dasgupta S, Baicu CF, et al. SPARC Production by Bone Marrow-Derived Cells Contributes to Myocardial Fibrosis in Pressure Overload. Am J Physiol Heart Circ Physiol (2021) 320 (2):H604-H12. doi: 10.1152/ajpheart.00552.2020

59. Dunkel J, Viitala M, Karikoski M, Rantakari P, Virtakoivu R, Elima K, et al. Enhanced Antibody Production in Clever-1/Stabilin-1-Deficient Mice. Front Immunol (2018) 9:2257. doi: 10.3389/fimmu.2018.02257
60. Schledzewski K, Geraud C, Arnold B, Wang S, Grone HJ, Kempf T, et al. Deficiency of Liver Sinusoidal Scavenger Receptors Stabilin-1 and -2 in Mice Causes Glomerulofibrotic Nephropathy via Impaired Hepatic Clearance of Noxious Blood Factors. J Clin Invest (2011) 121(2):703-14. doi: 10.1172/ JCI44740

61. Zhang Y, Zhang G, Liu Y, Chen R, Zhao D, McAlister V, et al. GDF15 Regulates Malat-1 Circular RNA and Inactivates NFkappaB Signaling Leading to Immune Tolerogenic DCs for Preventing Alloimmune Rejection in Heart Transplantation. Front Immunol (2018) 9:2407. doi: 10.3389/fimmu. 2018.02407

62. Wakchoure S, Swain TM, Hentunen TA, Bauskin AR, Brown DA, Breit SN, et al. Expression of Macrophage Inhibitory Cytokine-1 in Prostate Cancer Bone Metastases Induces Osteoclast Activation and Weight Loss. Prostate (2009) 69(6):652-61. doi: 10.1002/pros.20913

63. Hinoi E, Ochi H, Takarada T, Nakatani E, Iezaki T, Nakajima H, et al. Positive Regulation of Osteoclastic Differentiation by Growth Differentiation Factor 15 Upregulated in Osteocytic Cells Under Hypoxia. J Bone Miner Res (2012) 27 (4):938-49. doi: 10.1002/jbmr.1538

64. Westhrin M, Moen SH, Holien T, Mylin AK, Heickendorff L, Olsen OE, et al. Growth Differentiation Factor 15 (GDF15) Promotes Osteoclast Differentiation and Inhibits Osteoblast Differentiation and High Serum GDF15 Levels are Associated With Multiple Myeloma Bone Disease. Haematologica (2015) 100(12):e511-4. doi: 10.3324/haematol.2015.124511

65. Vanhara P, Lincova E, Kozubik A, Jurdic P, Soucek K, Smarda J. Growth/ differentiation Factor-15 Inhibits Differentiation Into Osteoclasts-a Novel Factor Involved in Control of Osteoclast Differentiation. Differentiation (2009) 78(4):213-22. doi: 10.1016/j.diff.2009.07.008

66. Chakravorty N, Hamlet S, Jaiprakash A, Crawford R, Oloyede A, Alfarsi M, et al. Pro-Osteogenic Topographical Cues Promote Early Activation of Osteoprogenitor Differentiation via Enhanced TGFbeta, Wnt, and Notch Signaling. Clin Oral Implants Res (2014) 25(4):475-86. doi: 10.1111/clr.12178

67. Khan MR, Donos N, Salih V, Brett PM. The Enhanced Modulation of Key Bone Matrix Components by Modified Titanium Implant Surfaces. Bone (2012) 50(1):1-8. doi: 10.1016/j.bone.2011.07.040

68. Eger M, Hiram-Bab S, Liron T, Sterer N, Carmi Y, Kohavi D, et al. Mechanism and Prevention of Titanium Particle-Induced Inflammation and Osteolysis. Front Immunol (2018) 9:2963. doi: 10.3389/fimmu.2018.02963

69. Hagstrom E, Held C, Stewart RA, Aylward PE, Budaj A, Cannon CP, et al. Growth Differentiation Factor 15 Predicts All-Cause Morbidity and Mortality in Stable Coronary Heart Disease. Clin Chem (2017) 63(1):325-33. doi: 10.1373/clinchem.2016.260570

70. Miller RJH, Teuteberg JJ, Hunt SA. Innovations in Ventricular Assist Devices for End-Stage Heart Failure. Annu Rev Med (2019) 70:33-44. doi: 10.1146/ annurev-med-041217-011015

Conflict of Interest: The authors declare that the research was conducted in the absence of any commercial or financial relationships that could be construed as a potential conflict of interest.

Publisher's Note: All claims expressed in this article are solely those of the authors and do not necessarily represent those of their affiliated organizations, or those of the publisher, the editors and the reviewers. Any product that may be evaluated in this article, or claim that may be made by its manufacturer, is not guaranteed or endorsed by the publisher.

Copyright (๑) 2021 Silva-Bermudez, Sevastyanova, Schmuttermaier, De La Torre, Schumacher, Klüter and Kzhyshkowska. This is an open-access article distributed under the terms of the Creative Commons Attribution License (CC BY). The use, distribution or reproduction in other forums is permitted, provided the original author(s) and the copyright owner(s) are credited and that the original publication in this journal is cited, in accordance with accepted academic practice. No use, distribution or reproduction is permitted which does not comply with these terms. 